\title{
Three-dimensional motion skeleton reconstruction algorithm for gymnastic dancing movements
}

\author{
Wenxian Fan*, \\ Department of Physical Education, Yuzhang Normal University, \\ Nanchang, 330000 \\ China \\ Yebing Zou, \\ Physical Education Department, Jiangxi University of Traditional Chinese Medicine, \\ Nanchang, 330004 \\ China
}

Received: June 20, 2021. Revised: December 16, 2021. Accepted: December 31, 2021. Published: January 3, 2022.

\begin{abstract}
Aiming at the problem of inaccurate matching results in the traditional three-dimensional reconstruction algorithm of gymnastic skeleton, a three-dimensional motion skeleton reconstruction algorithm of gymnastic dance action is proposed. Taking the center of gravity of the human body as the origin, the position of other nodes in the camera coordinate system relative to the center point of the human skeleton model is calculated, and the human skeleton data collection is completed through action division and posture feature calculation. Polynomial density is introduced into the integration of convolution surface, and the human body model of convolution surface is established according to convolution surface. By using the method of binary parameter matching, the accuracy of the matching results is improved, and the three-dimensional skeleton of gymnastic dance movement is reconstructed. The experimental results show that the fitting degree between the proposed method and the actual reconstruction result is $99.8 \%$, and the reconstruction result of this algorithm has high accuracy.
\end{abstract}

Keywords-Three-dimensional image, gymnastic dancing, skeleton reconstruction.

\section{INTRODUCTION}

$\mathrm{I}_{\mathrm{d}}^{\mathrm{N}}$ $\mathrm{N}$ recent years, as the vigorous development of gymnastic dancing, a higher requirement for the accuracy of movements in athlete competitions has been set [1]. Three-dimensional motion reconstruction is to recover the three-dimensional structure of the object from the image sequence. The three-dimensional structure is based on the camera, and its essence is to reconstruct the three-dimensional structure by using two or more two-dimensional images of the object. It is important for athletes to improve their experience and confidence by capturing and analyzing gymnasts' gymnastic dancing movements during the competition [2]. 3D motion skeleton reconstruction method has become the focus of research in the field of gymnastic dancing, and has attracted the attention of many experts and scholars.

Literature [3] proposes a three-dimensional reconstruction method of lung CT image based on graph theory segmentation, the improved minimum spanning tree method is used to segment the lung CT image. The marching cubes (MC) algorithm in surface rendering is used for $3 \mathrm{D}$ reconstruction, and the $3 \mathrm{D}$ display of lung is realized. The simulation results show that the improved minimum spanning tree algorithm is fast and effective. The simulation results are compared with those based on threshold segmentation. The results show that the improved algorithm can improve the efficiency and integrity of 3D reconstruction of lung CT image. At the same time, it can get better 3D reconstruction effect. This study can provide a strong basis for doctors' medical diagnosis. However, the algorithm in this method is more complex and error-prone, resulting in inaccurate reconstruction results. A method of 3D reconstruction of SRM section image based on VTK is proposed to model the 3D data field of section image and analyze the principle of various imaging algorithms [4]. In this 
paper, the cross-section image reconstruction subsystem and 3D visualization subsystem are designed based on modularization idea. Then, the implementation process of volume data is given, and the volume rendering and surface rendering are analyzed. Finally, the effectiveness of the system is verified by 180 SRM simulation film data. The results show that the image reconstruction effect by light projection algorithm is the best, and the size, the size and the shape of the image are the best The resolution and other indicators meet the design requirements, and the shape and location of defects are clearly visible; Contour volume rendering algorithm for image visualization. However, the $3 \mathrm{D}$ reconstruction time of this method is long.

In view of the above problems, a $3 \mathrm{D}$ motion skeleton reconstruction algorithm for gymnastics is proposed. Firstly, the human skeleton data is collected, and then the convolution surface is used to build the convolution surface human body model. Secondly, the binary parameter matching method is used to increase the precision of the matching results, and the three-dimensional skeleton reconstruction of gymnastics dance action is completed. Lastly, the validity of this method is verified by contrast experiments.

\section{3D Human MOTION MODEL}

Modeling of human body motion is a mathematical model to simulate human structure and motion. That is, the human body or human link is regarded as a rigid body with constant shape and size, or a particle with only mass but no size and shape, and the mathematical model of human structure and motion is established by using the theories and methods of mathematics, mechanics and physics, as well as the anatomical parameters of motion. When human is moving, the center of gravity is the most stable position in the whole body [5]. In this paper, the center of gravity is taken as the origin of the human body. The location of other nodes in the camera coordinate system relative to the center point of the human skeleton model is calculated by using:

$$
P^{(i)}=P_{c}^{(i)}-P_{c}^{(0)}
$$

where $i=1,2 \ldots, P_{c}^{(i)}$ are the coordinates of the node $i$ in the camera coordinate system, $P_{c}^{(0)}$ are the coordinates of the center point of the skeleton in the camera coordinate system.

Convolution surface is defined by the skeleton consisting of the space points. The convolution surface is essentially an isosurface of the scalar field defined by the skeleton through convolution. Any point $(x, y, z)$ on a surface satisfies the implicit equation, which is given by:

$$
F(x, y, z)-T=0
$$

where $T$ is a threshold, $F(x, y, z)$ is the field function generated by the whole skeleton, which is obtained by the integral of the product of the geometric skeleton function $\vec{r}$ and the potential function of each point, $f(\vec{r})$ is an arbitrary point on the surface, then:

$$
f(\vec{r})=\frac{1}{\left(1+s^{2}\|\vec{r}\|^{2}\right)^{2}}
$$

where $S$ is the radius adjustment parameter, which can dominate the breadth of the kernel. A polynomial density is introduced into the integral of convolution surface, then:

$$
\frac{\int_{0}^{t}\left(\sum_{i=0}^{n} q_{i} t^{i}\right)}{\left(1+s^{2}\|\vec{r}\|^{2}\right)^{2}} d t-T=0
$$

In the formula, $\mathrm{t}$ denotes the time to construct a mannequin. The first two coefficients $q_{0}$ and $q_{1}$ of the polynomial are used to adjust the different shapes of the convolution surface. $n$ is the number of the fold line segments. When constructing the human body model, the human skeleton is divided into 32 fold line segments.

In the optimization process, in order to reduce the number of local optimal solutions, a distance constraint between joint and human image region is given [6]. The intuitive meaning is to make the projection of the joint points of the limbs and head as close as possible to the point set after the skeletonization of the real image. First, the human body is skeletonized to obtain the point set $Q . P$ is the projection set of joint points. Define the distance of an arbitrary point $\vec{p}_{i} \in P$ and $Q$ is $D\left(p_{i}, Q\right)=\min _{j}\left\|\vec{p}_{i}-\vec{q}_{j}\right\|^{2} \cdot \forall \vec{q}_{j} \in Q$, then the nonlinear constraint condition is expressed as:

$$
G(X)=\sum_{i} D\left(p_{i}, Q\right)<T
$$

\section{BINARY PARAMETER METHOD}

The problem of skeleton reconstruction is simplified as: The name $\left\{l_{i}\right\}$ of each part of the human body is allocated to each part $\left\{c_{i}\right\}$ of the human body in the gymnastic dancing image, and then a unitary constraint parameter $f_{k}$ is used to determine whether the allocation is feasible [7]. The constraint parameter $f_{k}$ is expressed in the form of Gaussian distribution $\left(\mu_{k}, \sigma_{k}\right)$, gaussian distribution refers to normal distribution, if random variable $\mathrm{x}$ obeys a mathematical expectation, it is $\mu$. The variance is $\sigma 2$, denoted as $\mathrm{n}(\mu, \sigma 2)$. The probability density function is the expected value of normal distribution $\mu$. Its position and standard deviation are determined $\sigma$. It determines the magnitude of the distribution. Then:

$\bar{f}_{k}=\frac{\left(f_{k}-\mu_{k}\right)^{2}}{\sigma_{k}^{2}}$

The problem of joint determination is quantified and converted to binary parameter [8]. A binary parameter vector $x$ is used to represent the allocation function $\pi$. In this way, the joint decision problem is transformed into a binary parameter problem [9]. The binary parameter equation is given by:

$$
\left\{\begin{array}{l}
\min Q(x)=x^{\prime} H x+c^{\prime} \\
\text { subject to } A x=b_{i}, \quad x \in\{0,1\}^{n}
\end{array}\right.
$$


where $H$ is the parallel area found in the gymnastic dancing movement image, and given by:

$$
H_{i, j}=\sum_{k} \bar{f}[l(i), l(j), c(i), c(j)]
$$

When the set binary parameter makes (7) minimum, Equation (8) also obtains the minimum value correspondingly. Finally, $2 \mathrm{D}$ image reconstruction of gymnastic dancing movements is achieved.

\section{EXPERIMENTAL RESULTS AND ANALYSIS}

In order to verify the effectiveness of the proposed algorithm, simulation experiments are executed. The experimental environment is: Windows 7 operating system, the main frequency of processor is $3.40 \mathrm{GHz}$, CPU Intel (R) core (TM) i7-3770 m, and 4G memory. MATLAB r2010a is used to realize algorithm programming. There are 10000 sample sets, 5000 training samples and 5000 test samples. 50 groups of experiments are carried out with 100 data each time.

In this paper, taking the images of gymnastic women's team final in the 2007 East Asian Games as an example, the 3D image skeleton reconstruction of gymnastic dancing movements is carried out. According to the Euler angle, the human body is marked in the direction perpendicular to the camera with the center of gravity of the human body as the origin. The position of each joint in the image is calibrated to make the projection of the connection point between the limbs and the head as close as possible to the set connection point, and the basic model of human skeleton is obtained. Then the Gaussian parameter is introduced into the basic model and the Gaussian model is used as the standard human model, as shown in Fig. 1.

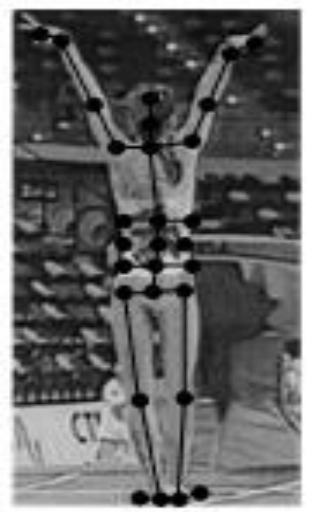

Fig. 1 human body model

The gymnastic images selected in this experiment contain stretching movements of run-up and forward somersault. The run-up is divided into two stages. (1) The first pull stage, that is, before the run-up, the foot touches the surface of the ground. (2) Inertial forward stage, that is, the whole body moves away from the ground, and the body moves forward with inertia.

The image of gymnastics can be placed on a 2D plane, and the coordinates of human joints can be determined by motion division and pose feature. The trajectory of coordinate points is connected according to Gaussian model to obtain the 2D model of human body, as shown in Fig. 2.
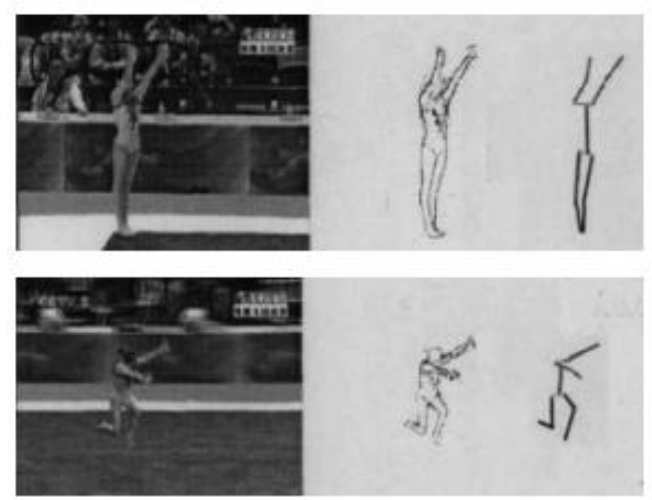

Fig. 2 2D model

In order to make the result obtained with the proposed method more accurate, the binary parameter method is used to process the deviations in two steps.

Step 1: Assume the motion of human body is a linear motion. The wrong joint points in the process are deleted, and the binary parameter method is used to complement the deleted error nodes.

The $x$-axis coordinates of the left knee joint of the tester in gymnastic dancing image are taken as example, as shown in Fig. 3.

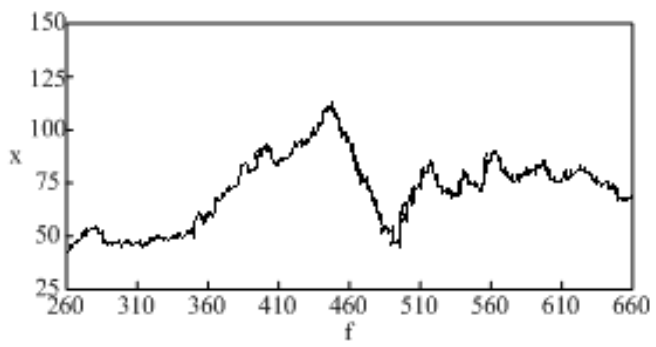

Fig. 3 change of $\mathrm{x}$-axis coordinates of left knee joint of gymnast with time

From Fig. 3, it can be seen that, there is an obvious error in the $\mathrm{x}$-axis coordinates of the 440-527 frame. The error of this time period is deleted and the deleted error nodes are complemented by using the binary parameter method, as shown in Fig. 4.

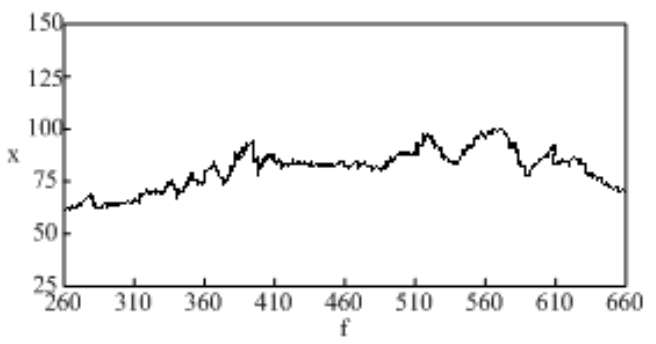

Fig. 4 the complemented part by using the binary parameter method

Step 2: The obtained joint is substituted into the binary parameter model, and the inapplicable joint position coordinates are deleted. Then execute the step 1.

The more repeated operations in these two steps, the more accurate the result of reconstruction. In this way, multiple operations are made to make the result of the proposed method more precise.

Massive 3D human skeleton model with Gaussian constraint 
ratio is built and shown in Fig. 5.

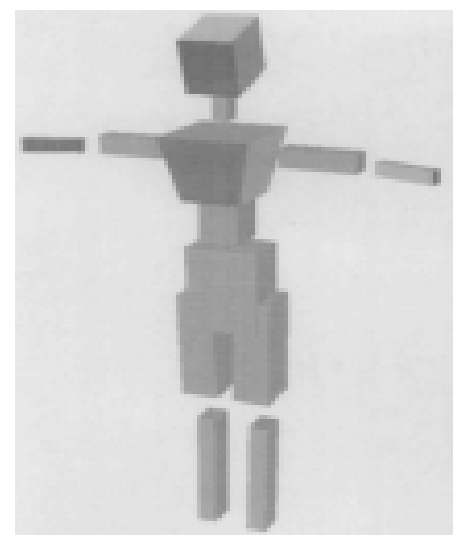

Fig. 5 3D human skeleton model with Gaussian constraint ratio

By using the length of the body part in the image and the length of the body part of the Gaussian model, the relative displacement of the joint and its adjacent joint on the z-axis is obtained by using the binary parameter method. Two points with the same relative displacement are found on the z-axis. These two points are substituted into the binary parameter equation and joint position that does not meet the requirements is deleted. Then the final transformation results are obtained and the $3 \mathrm{D}$ skeleton reconstruction of gymnastic dancing movements is achieved, as shown in Fig. 6.

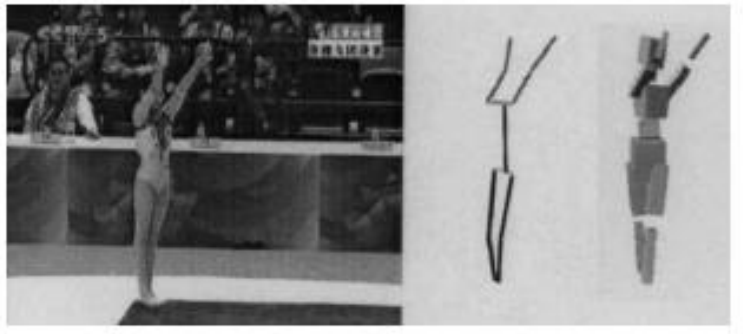

Fig. $63 \mathrm{D}$ skeleton

In order to verify the effectiveness of this method, the precise of the reconstruction results of the three-dimensional image of the gymnastic movement skeleton of the methods in this paper, literature [3] and literature [4] are compared and analyzed, and the comparison results are shown in Fig. 7.

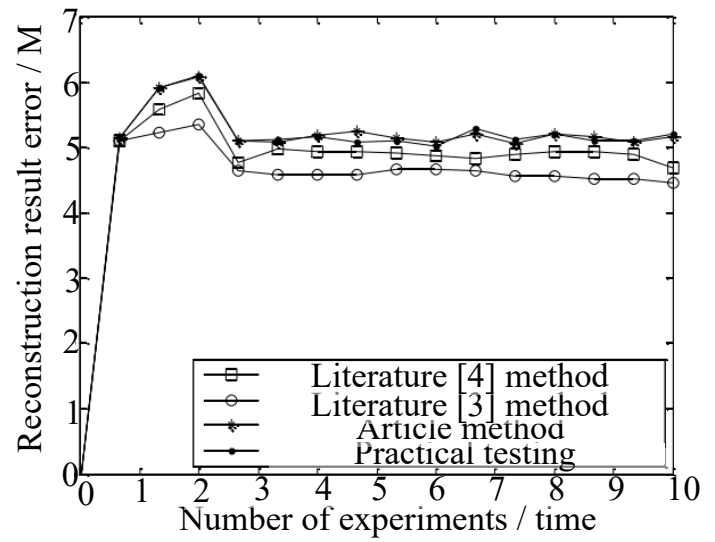

Fig. 7 accuracy contrast of reconstruction consequence

In conformity with Fig. 7, the fitting degree among the reconstruction consequences of the three-dimensional image of gymnastic movement skeleton of the method in this paper and the practical reconstruction consequences is $99.8 \%$, when the reconstruction consequences of the three-dimensional image of gymnastic movement skeleton of the methods in document [3] and document [4] are totally different from the practical reconstruction consequences, which shows that the technique in this paper has high accuracy of the reconstruction consequences of the three-dimensional image of gymnastic movement skeleton. This is because this method uses the binary parameter matching method, which improves the accuracy of the matching results.

To further verify the effectiveness of the proposed method, the three-dimensional image reconstruction time of gymnastics movement skeleton of the technique in this paper, the technique in document [3] and the technique in document [4] are contrasted and analyzed, and the contrastion results are shown in Fig. 8.

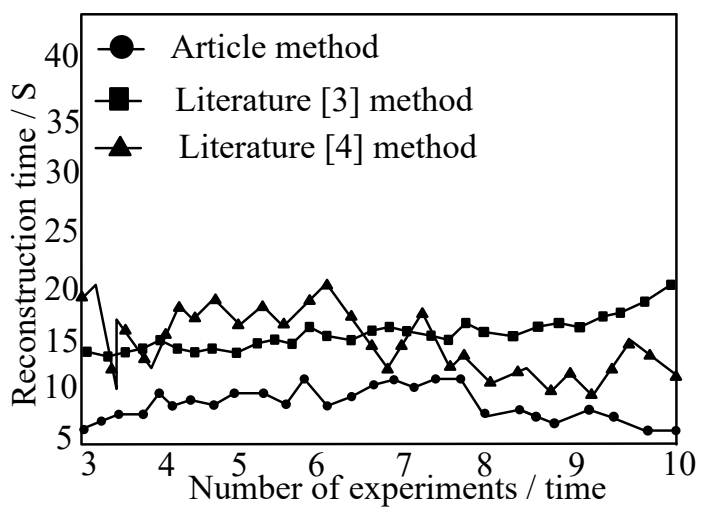

Fig. 8 contrast of reconstruction time

According to Fig. 8, the reconstruction time of three-dimensional image of gymnastic movement skeleton in this technique is shorter than that in document [3] and document [4]. This is because the method reduces the number of local optimal solutions, gives the distance constraints between the joint and the human image region, and shortens the reconstruction time.

\section{CONCLUSION}

The matching algorithm in the traditional 3D skeleton reconstruction method is more complex, resulting in inaccuracy of skeleton reconstruction results. In this paper, a 3D image motion skeleton reconstruction method for gymnastic dancing movements is proposed.

(1) The purpose of the research is to reconstruct $3 \mathrm{D}$ skeleton in gymnastic dancing movement image and improve the movement quality in gymnastic dancing.

(2) The fitting degree of the 3D image reconstruction results of the gymnastics skeleton obtained by this method and the actual reconstruction results is $99.8 \%$, which has high precision and shortens the reconstruction time of the $3 \mathrm{D}$ image of gymnastics. At the same time, it also verifies the validity and feasibility of this method.

(3) Due to the clothing on the subject and the different skeleton structure between the subject and the subject, the test 
results will be affected. Therefore, in the future research, the above situation will be taken into account, and the performance of this method will be further verified by human motion capture simulation technology.

\section{REFERENCES}

[1] T. Ekeberg, M. Svenda, and C. Abergel, "Three-dimensional reconstruction of the giant mimivirus particle with an x-ray free-electron laser," Physical Review Letters, vol. 114, no. 9, pp. 098102, 2015.

[2] R. Dahl, S. Larsen, and T. L. Dohlmann, "Three-dimensional reconstruction of the human skeletal muscle mitochondrial network as a tool to assess mitochondrial content and structural organization," Acta Physiologica, vol. 213, no. 1, pp. 135-137, 2015.

[3] B. X. Cui, J. Tian, and Y. Duan, "Three-dimensional reconstruction of lung CT images based on graph theory segmentation," Journal of Shenyang University of Technology, vol. 37, no. 6, pp. 667-672, 2015.

[4] C. Q. Shen, and J. Gao, "Design of SRM cross-section image 3D reconstruction system based on visualization toolkit," Computer Simulation, vol. 33, no. 3, pp. 90-94, 2016.

[5] D. Kim, and O. Sahin, "Imaging and three-dimensional reconstruction of chemical groups inside a protein complex using atomic force microscopy," Nature Nanotechnology, vol. 10, no. 3, pp. 264-269, 2015.

[6] J. Cabrera, M. Barcala, and I. Argandacarreras, "Phenotyping nematode feeding sites: Three-dimensional reconstruction and volumetric measurements of giant cells induced by root-knot nematodes in Arabidopsis," New Phytologist, vol. 206, no. 2, pp. 868-80, 2015.

[7] M. Rafeek, M. Hazem, and A. El-Bakry, "Converting 2D-medical image files "DICOM" into 3D- models, based on image processing, and analysing their results with python programming," WSEAS Transactions on Computers, vol. 19, no. 2, pp. 10-20, 2020.

[8] H. Luqman, and I. Muhammad, "Implementation of discrete wavelet transform on movement images and recognition by artificial neural network algorithm," WSEAS Transactions on Signal Processing, vol. 15, no. 18, pp. 149-154, 2019.

[9] S. Siranush, and H. Anna, "Neural network effectiveness evaluation while the intersection of images in the receptor field," WSEAS Transactions on Information Science and Applications, vol. 17, no. 21, pp. 172-176, 2020.

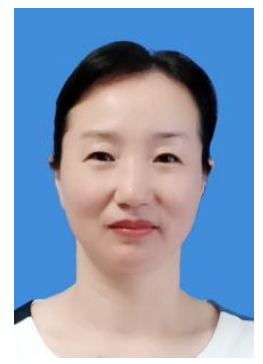

Wenxian Fan, born in December 1975, female, teacher, university lecturer. She graduated from Jiangxi Normal University with a bachelor's degree in physical education in July 2002, and graduated with a master's degree in software from Huazhong University of Science and Technology in July 2011. Now She works as a physical education teacher at Yuzhang Normal University in Jiangxi Province. Her academic research direction is physical education. She has published two core papers, participated in 5 scientific research projects, and 12 provincial papers. The title of this paper is: 3D image motion bone reconstruction algorithm for gymnastics and dance movements.

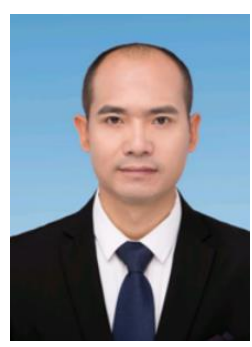

Yebing Zou, male, born in March 1976, teacher, associate professor of colleges and universities, postgraduate. In July 2004, he graduated from Qufu Normal University with a bachelor's degree in physical education, and in July 2009, he graduated from Jiangxi Normal University with a master's degree. Now his work unit is a physical education teacher at Jiangxi University of Traditional Chinese Medicine. His academic research direction is physical education and sports rehabilitation. He has published 16 papers including 5 core papers, and participated in 9 scientific research projects. The title of this paper is: 3D image motion bone reconstruction algorithm for gymnastics and dance movements.

Wenxian Fan and Yebing Zou proposed a three-dimensional motion skeleton reconstruction algorithm for gymnastics and dance movements. Wenxian Fan takes the center of gravity of the human body as the origin, calculates the position of other nodes in the camera coordinate system relative to the center point of the human skeleton model, and completes the collection of human skeleton data through action division and pose feature calculation. Yebing Zou introduced polynomial density into the integration of convolution surface, and established convolution surface manikin according to convolution surface. Wenxian Fan and Yebing Zou conducted research experiments, recorded the actual reconstruction data, and created manuscripts. All authors read and approved the final manuscript.

\section{Creative Commons Attribution License 4.0 (Attribution 4.0 International, CC BY 4.0)}

This article is published under the terms of the Creative Commons Attribution License 4.0 https://creativecommons.org/licenses/by/4.0/deed.en_US 